\title{
Intramolecular Charge Transfer Processes in Confined Systems. Nile Red in Reverse Micelles $^{\dagger}$
}

\author{
Anindya Datta, Debabrata Mandal, Samir Kumar Pal, and Kankan Bhattacharyya* \\ Department of Physical Chemistry, Indian Association for the Cultivation of Science, Jadavpur, \\ Calcutta 700 032, India
}

Received: May 9, 1997; In Final Form: August 18, $1997^{\circledR}$

\begin{abstract}
Intramolecular charge transfer (ICT) processes of the neutral fluorescence probe, nile red, I, confined in the water pool of aerosol-OT (AOT) reverse micelle in $n$-heptane, is studied using picosecond emission spectroscopy. Utilizing the solvatochromism of nile red, only those probe molecules inside the reverse micelle are selectively excited. It is observed that while in aqueous solutions the lifetime $\left(\tau_{\mathrm{f}}\right)$ of nile red is $650 \mathrm{ps}$, inside the reverse micelles $\tau_{\mathrm{f}}$ increases to $3.73 \mathrm{~ns}$ in reverse micelle and to $2.06 \mathrm{~ns}$ at the highest water content of the microemulsion $\left(w_{\mathrm{o}}=32\right)$. With increase in the water-to-surfactant ratio, $w_{\mathrm{o}}$, as the water pool swells in size, the lifetime and quantum yield of emission decrease and the rate of the ICT process of nile red increases. However, the magnitude of the change (at most 8 times) in the rate of the ICT process of nile red compared to that of ordinary water is much smaller than the several thousandfold decrease observed in the solvation dynamics of water in the water pool relative to bulk water. It is proposed that while the solvation dynamics in the water pool is governed by the dielectric relaxation time, dynamics of the ICT process is controlled by the static polarity of the medium.
\end{abstract}

\section{Introduction}

The water molecules bound to different biological and organized assemblies play a crucial role in many natural processes. The interplay between the water-water and the water-organic interactions control the structure, function, and dynamics of many biological systems such as proteins, biomembranes, vesicles, etc. Since most natural processes occur in such confined or organized systems, in recent years several new experimental $^{1-7}$ and theoretical techniques ${ }^{2,8,9}$ have been used to study such systems. Several groups have used surface second harmonic and surface sum frequency generation ${ }^{1}$ and picosecond total internal reflection spectroscopy ${ }^{3}$ to study the structure and the dynamics at various interfaces. The time-resolved Stokes shift has also been used by several other groups to study solvation dynamics in various organized assemblies such as cyclodextrins, ${ }^{4,8 a}$ reverse micelles, ${ }^{5,6 a, b}$ and micelles. ${ }^{6 c}$ The results of the dynamic Stokes shift studies indicate that while ordinary water molecules relax in the subpicosecond time scale, inside these organized assemblies the solvation dynamics becomes several thousand times slower and occurs in the nanosecond time scale. This observation is consistent with the dielectric relaxation and the pulsed NMR studies. ${ }^{7}$ These studies demonstrate that while dielectric relaxation time of water is about $10 \mathrm{ps}$, in aqueous proteins the dielectric relaxation is almost universally bimodal with one component of about $10 \mathrm{ps}$ and a 1000-fold slower component of $10 \mathrm{~ns}$. This suggests that the water molecules bound to biological and organized assemblies are much slower than ordinary water. The early workers proposed that in such systems there are two kinds of water molecules, "bound" and "free", relaxing in the nano- and picosecond time scale, respectively. ${ }^{7}$ However, a more recent model envisages an equilibrium between the bound and the free water in biological systems which results in the bimodal dielectric relaxation. . $^{8}$

* Corresponding author. e-mail: pckb@iacs.ernet.in; Fax: (91)-33-4732805.

†Dedicated to Professor M. V. George on his 70th birthday.

${ }^{\otimes}$ Abstract published in Advance ACS Abstracts, November 1, 1997.
The so-called reverse micelles formed by surfactant molecules in hydrocarbon solvents with their polar headgroups pointing inward are simple yet interesting models of biological membranes and the water molecules confined in biological systems. ${ }^{2 \mathrm{~b}, 5,6 \mathrm{a}, \mathrm{b}, 14-16}$ In hydrocarbon solvents the surfactant aerosolOT (sodium bis(2-ethylhexyl) sulfosuccinate, AOT) forms reverse micelles at concentrations above $1 \mathrm{mM}$ with average aggregation number 20 and radius $15 \AA \AA^{15,16}$ On addition of water to a solution of AOT in hydrocarbon solvents, a microemulsion is formed which is basically a nanometer sized water droplet surrounded by a layer of the surfactant molecule. In $n$-heptane the radius $\left(r_{\mathrm{w}}\right)$ of such a water pool is about $2 w_{\mathrm{o}}$ (in angstroms) where $w_{\mathrm{o}}$ is the number ratio of the water to the surfactant molecules. ${ }^{16 \mathrm{~d}}$ In such a water pool the water molecules at the peripheries of the pool are strongly held by the polar or ionic headgroups of the surfactants and are thus "bound", while those at the center of the pool are relatively "free". The amount of free and bound water molecules present in such a microemulsion has been estimated and tabulated by many workers..$^{2 b, 15}$ Recently, Bright et al. ${ }^{5}$ and our group ${ }^{6 a, b}$ separately reported that in such a water pool of a microemulsion the solvent relaxation times are $2-10 \mathrm{~ns}$, which is several thousand times slower than the subpicosecond relaxation time in ordinary water reported by Fleming et al. ${ }^{4}$ Robinson et al. ${ }^{2 b}$ studied 1-anilino-8-naphthalenesulfonate (ANS) in AOT reverse micelles and reported that while the lifetime of ANS is 280 ps in ordinary water, in the reverse micelles it increases markedly, and from the biexponential decays the average lifetime $\langle\tau\rangle=$ $a_{1} \tau_{1}+a_{2} \tau_{2}$ is estimated to be as large as $3.6 \mathrm{~ns}$ at $w_{\mathrm{o}}=4$. It may be recalled that a nanosecond component in the fluorescence decay of ANS is also observed at the heptane-water interface using picosecond total internal reflection spectroscopy by Bessho et al. ${ }^{3 a}$

In the present work we report on the intramolecular charge transfer process in nile red in AOT reverse micelles. Nile red, a neutral molecule, has recently been shown to be an excellent fluorescence probe for biological systems. ${ }^{10}$ We have shown earlier ${ }^{11}$ that the nonradiative rate of nile red increases expo- 
nentially with the polarity parameter $E_{\mathrm{T}}(30)$ of the medium and have assigned this nonradiative process to the polarity-dependent twisted intramolecular charge transfer (ICT) process. ${ }^{12,13}$ The main advantage of nile red as a probe for such systems is the fact that the absorption maximum of nile red strongly depends on the polarity of the medium. $\lambda_{\max }^{\mathrm{abs}}$ of the lowest energy band of nile red shifts from $510 \mathrm{~nm}$ in $n$-heptane to $550 \mathrm{~nm}$ in water. Thus, at $\lambda>540 \mathrm{~nm}$, while nile red in hydrocarbon medium absorbs negligibly, those in the polar region absorb quite strongly. Thus, as we will show in the following sections, in a microemulsion at $\lambda>540 \mathrm{~nm}$ one can selectively excite and study only those nile red molecules that are encapsulated in the polar water pool of the reverse micelles, without affecting the nile red molecules abundant in the bulk $n$-heptane. Our intention is to see how the several thousand times slower water molecules present in such a water pool affect the ICT process of nile red (I).<smiles>CCCCNc1ccc2nc3c4ccccc4c(=O)cc-3oc2c1</smiles>

I

\section{Experimental Section}

Nile red (Aldrich) was purified by thin-layer chromatography using spectroscopy grade chloroform as eluent. Purification and drying of sodium bis(2-ethylhexyl) sulfosuccinate (AOT, Aldrich) is described elsewhere. ${ }^{6 \mathrm{~b}, 15 \mathrm{~b}}$ To nile red dissolved in freshly distilled $n$-heptane was added solid AOT. Then a requisite amount of water was added using a microliter syringe. An aqueous solution of nile red was prepared following the reported procedure. ${ }^{10}$ Wolff et al. reported that in aqueous solution nile red decomposes with a half-life of $\approx 20 \mathrm{~min}^{10}$ Thus, all measurements for nile red in water were done within 15 min after making the solution. Still a reasonably good quantum yield $\left(\phi_{\mathrm{f}}\right)$ value in aqueous solution is obtained as the calculated $E_{\mathrm{T}}(30)$ value of water in this work is 61.3 , which is close to the reported $E_{\mathrm{T}}(30)$ of water (63.6). ${ }^{19}$ In the reverse micelle and microemulsions, however, nile red was found to be stable for many hours. For $\phi_{\mathrm{f}}$ measurements as standard we used Rhodamine $6 \mathrm{G}$ in methanol ${ }^{20}$ for excitation at $490 \mathrm{~nm}$ and nile red in methanol ${ }^{11}$ for excitation at 540-570 nm. For lifetime measurements the sample was excited with the fundamental of a cavity dumped synchronously pumped Rhodamine 6G dye laser (Coherent 702) pumped by a CW mode-locked Nd:YAG laser (Coherent Antares 76S). The fluorescence was detected at magic angle polarization by a Hamamatsu MCP photomultiplier (2809U). ${ }^{6 \mathrm{~b}}$ The time resolution of this setup is $\approx 100 \mathrm{ps}$. Steady-state absorption and emission spectra were recorded on a JASCO 7850 spectrophotometer and a PerkinElmer MPF 44B spectrofluorimeter, respectively.

\section{Results and Discussion}

3.1. Steady-State Absorption Spectra. In $n$-heptane nile red exhibits two prominent absorption peaks in the visible region at 510 and $490 \mathrm{~nm}$ (Figure 1a) with negligible absorption above $540 \mathrm{~nm}$. On addition of $0.09 \mathrm{M}$ AOT to $n$-heptane, the absorbance of nile red at $\lambda>520 \mathrm{~nm}$ increases and that in the 490-500 nm region decreases (Figure 1a). The difference spectrum (Figure $1 \mathrm{~b}$ ) between nile red in $n$-heptane with 0.09 M AOT and the same without AOT exhibits a distinct peak at $533 \mathrm{~nm}$ and a trough at around $490 \mathrm{~nm}$. The $533 \mathrm{~nm}$ peak in
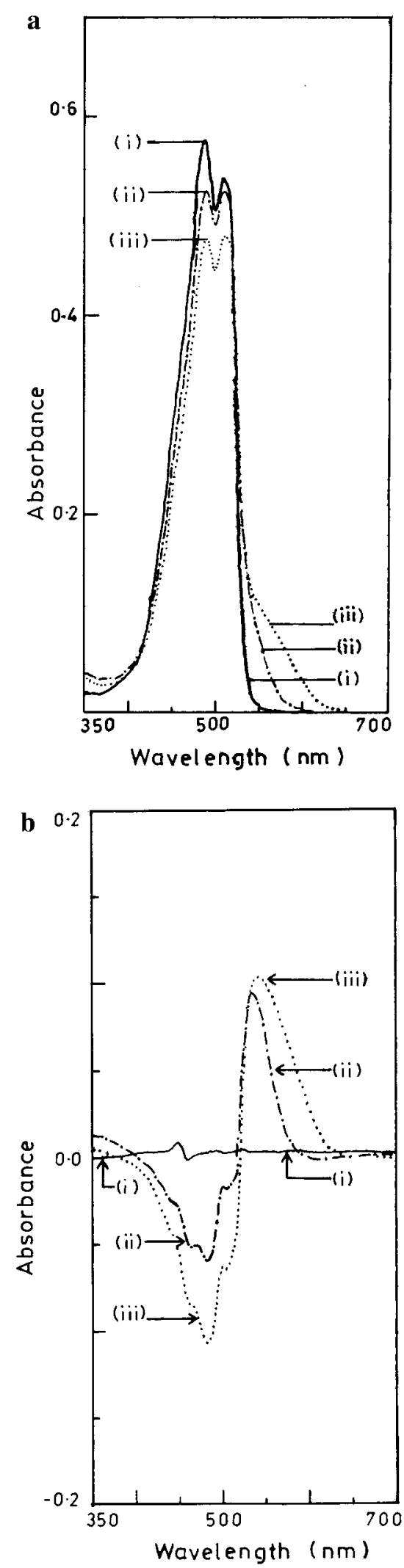

Figure 1. (a) Absorption spectrum of nile red in (i) $n$-heptane alone (-), (ii) $0.09 \mathrm{M}$ AOT in $n$-heptane, $w_{\mathrm{o}}=0(-\cdot-)$, and (iii) $0.09 \mathrm{M}$ AOT in $n$-heptane, $w_{\mathrm{o}}=32(\cdots)$. (b) Difference spectra between nile red in $n$-heptane with that of (i) nile red in $n$-heptane without AOT $(-)$, (ii) nile red in $0.09 \mathrm{M} \mathrm{AOT}, w_{\mathrm{o}}=0(-\cdot-)$, and (iii) nile red in $0.09 \mathrm{M} \mathrm{AOT}, w_{\mathrm{o}}=32(\cdots)$.

the difference spectrum is assigned to those nile red molecules inside the reverse micelles while the trough in the 490-500 $\mathrm{nm}$ region corresponds to depletion of nile red in bulk $n$-heptane due to the migration of nile red molecules from bulk $n$-heptane 

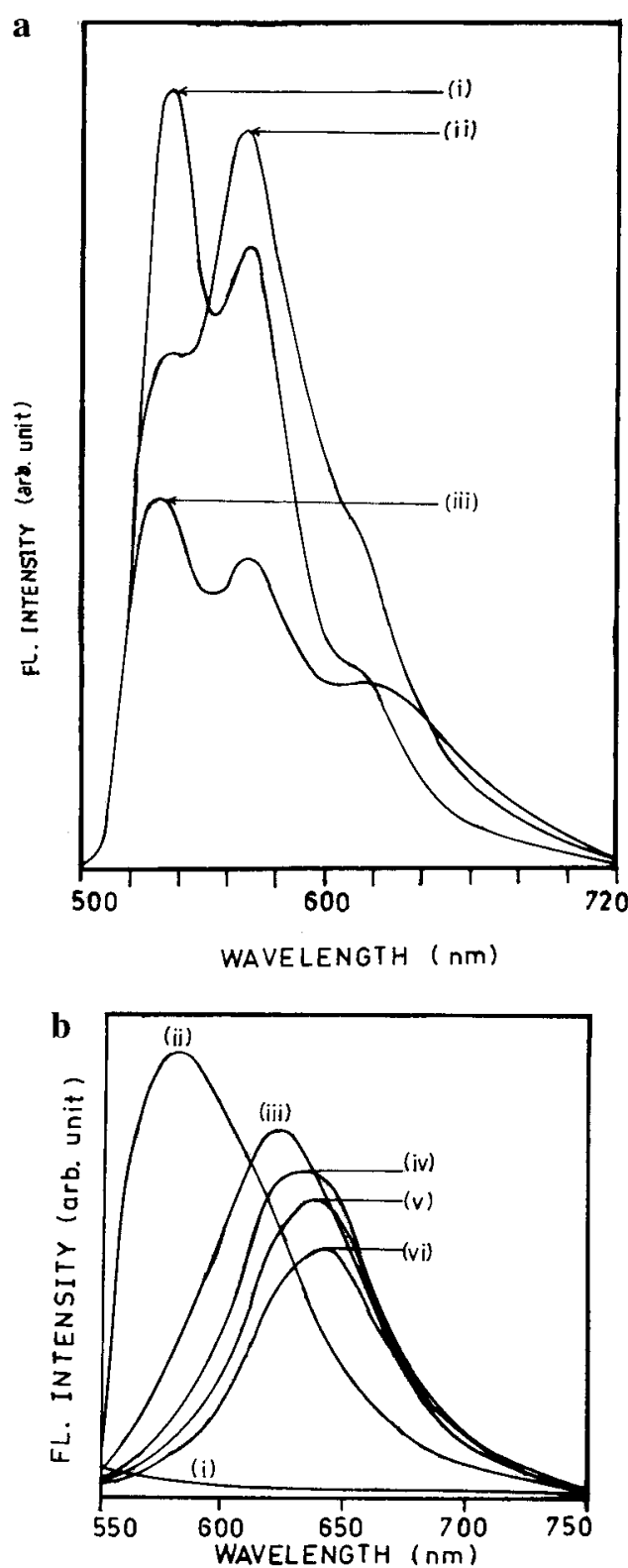

Figure 2. (a) Emission spectra of nile red, $\lambda_{\mathrm{ex}}=490 \mathrm{~nm}$, in (i) nile red in $n$-heptane without AOT, (ii) nile red in $0.09 \mathrm{M} \mathrm{AOT,} w_{\mathrm{o}}=0$, and (iii) nile red in $0.09 \mathrm{M}$ AOT, $w_{\mathrm{o}}=32$. (b) Emission spectra of nile red, $\lambda_{\mathrm{ex}}=550 \mathrm{~nm}$, in (i) nile red in $n$-heptane without AOT, (ii) nile red in $0.09 \mathrm{M} \mathrm{AOT}, w_{\mathrm{o}}=0$, (iii) $w_{\mathrm{o}}=4$, (iv) $w_{\mathrm{o}}=8$, (v) $w_{\mathrm{o}}=$ 16 , and (vi) $w_{\mathrm{o}}=32$.

to the interior of the reverse micelles. On addition of water to the $0.09 \mathrm{M}$ AOT solution in $n$-heptane the absorbance at $\lambda>$ $520 \mathrm{~nm}$ increases further (Figure 1a), and the peak in the difference spectrum, due to the nile red molecules in the water pool, becomes more prominent and shifts toward the red to 540 nm (Figure 1b).

3.2. Steady-State Emission Spectra. In $n$-heptane on excitation at around $500 \mathrm{~nm}$ nile red exhibits intense emission (quantum yield, $\phi_{\mathrm{f}}=0.48$ ) with two emission peaks at 538 and $568 \mathrm{~nm}$ and a shoulder at $620 \mathrm{~nm}$ (Figure 2a). However, when nile red in $n$-heptane is excited at wavelengths greater than 540 $\mathrm{nm}$, negligible emission is observed (Figure $2 \mathrm{~b}$ ). On addition of $0.09 \mathrm{M}$ AOT to the $n$-heptane solution of nile red for excitation in the $490-500 \mathrm{~nm}$ region, the intensity of the emission peak at $538 \mathrm{~nm}$ decreases while the intensity at the red end increases (Figure 2a). When excited at $\lambda>540 \mathrm{~nm}$, nile red in 0.09 M AOT in $n$-heptane displays a distinct emission peak at $580 \mathrm{~nm}$ (Figure 2b). Since at $\lambda>540 \mathrm{~nm}$ only those nile red molecules that are inside the reverse micelles absorb and since the $580 \mathrm{~nm}$ emission band is absent in the emission spectrum of nile red in $n$-heptane without AOT, this peak is assigned to those nile red molecules inside the reverse micelle of AOT. On addition of water to the $0.09 \mathrm{M}$ AOT solution, for excitation in the 490-500 $\mathrm{nm}$ region a further decrease in the intensity of the emission peaks at 538 and $568 \mathrm{~nm}$ is noticed. Excitation of the nile red solution in AOT $/ n$-heptane in the presence of water at wavelengths between 540 and $570 \mathrm{~nm}$ results in a single broad emission band with maximum at around $625 \mathrm{~nm}$ at $w_{\mathrm{o}}=4$ which gradually red shifts to $640 \mathrm{~nm}$ at $w_{\mathrm{o}}$ $\geqslant 16$ (Figure $2 \mathrm{~b}$ ). The $640 \mathrm{~nm}$ emission band, which is very much red-shifted from that of nile red in $n$-heptane without AOT and also from that of nile red in $0.09 \mathrm{M}$ AOT solution, is obviously assigned to the water pool of the microemulsion. The $\phi_{\mathrm{f}}, \lambda_{\max }^{\mathrm{em}}$, and radiative $\left(k_{\mathrm{r}}\right)$ and nonradiative rates $\left(k_{\mathrm{nr}}\right)$ of nile red in AOT $-n$-heptane microemulsion and also those in water are summarized in Table 1.

3.3. Time-Resolved Studies. In the time-resolved studies the samples were excited at $570 \mathrm{~nm}$ to excite selectively only those nile red molecules inside the reverse micellar aggregates. In aqueous solution nile red exhibits a single-exponential decay with lifetime 650 ps (Figure 3a). In reverse micelles, however, the decay of nile red becomes much longer and multiexponential (Figure 3b). Obviously extracting meaningful rate constants in such a heterogeneous system is difficult. Robinson et al. pointed out that if the diffusion coefficient $(D)$ of the probe in the water pool is the same as that of an aromatic molecule in ordinary water $\left(0.05 \AA^{2} \mathrm{ps}^{-1}\right)$, the probe molecule moves about $10 \AA / n s$ in a direction normal to the surface $\left(\left\langle z^{2}\right\rangle=2 D\langle t\rangle\right)$. ${ }^{2 b}$ Thus, the probe, nile red, molecules pass through several water layers within the observed lifetime of a few nanoseconds. Evidently the multiexponential decay originates from the different polarity regions experienced by the probe molecule within its lifetime. If the diffusion coefficient inside the water pool is slower by 1 order of magnitude compared to that of ordinary aqueous solution, every nile red molecule would be more or less confined to one layer of water molecules. However, even then there is the possibility of different nile red molecules in different polarity regions being excited simultaneously. That superposition of many such decays of slightly different lifetimes looks like a biexponential decay is discussed by many workers. ${ }^{2 b, 17}$ Instead of placing too much emphasis on the magnitude of individual decay constants in such biexponential decays, we have considered only the average lifetime $\langle\tau\rangle$ defined by $a_{1} \tau_{1}+a_{2} \tau_{2}$. The data in Table 2 indicate that with increase in the water content the average lifetime of nile red in the microemulsion decreases from $3.73 \mathrm{~ns}$ at $w_{\mathrm{o}}=$ 0 to $2.06 \mathrm{~ns}$ at $w_{\mathrm{o}}=32$. From the observed $\phi_{\mathrm{f}}$ and $\tau_{\mathrm{f}}$ the nonradiative rate constants $\left(k_{\mathrm{nr}}\right)$ are obtained as explained elsewhere. ${ }^{11}$ We had shown earlier that $\ln \left(k_{\mathrm{nr}}\right)$ of nile red is a linear function of $E_{\mathrm{T}}(30)$ of the medium. ${ }^{11}$ Using the $k_{\mathrm{nr}}$ values of nile red in the present work and the fitting parameters for the data of $k_{\mathrm{nr}}$ and $E_{\mathrm{T}}(30)$ in ref 11 , the $E_{\mathrm{T}}(30)$ of the microemulsions is estimated. The data are tabulated in Table 1. It is readily seen that the $E_{\mathrm{T}}(30)$ of the microemulsions obtained in this way is close to those reported in the literature. ${ }^{18}$

The results of the time-resolved studies indicate that while the solvation dynamics slows down several thousand times inside the reverse micelles, $5,6 a, b$ the dynamics of the ICT processes of nile red (i.e., $k_{\mathrm{nr}}$ ) is slowed by at most 8 times inside the water pool (at $\left.w_{\mathrm{o}}=4\right)$ compared to that of ordinary bulk water. The interior of the water pools differs in two ways from ordinary bulk water. ${ }^{6 a}$ First, the dielectric relaxation time $\left(\tau_{\mathrm{D}}\right)$ indicates a bimodal behavior with one component of 10 
TABLE 1: Emission Characteristics of Nile Red in 0.09 M AOT in $n$-Heptane

\begin{tabular}{|c|c|c|c|c|c|c|c|c|}
\hline medium & $r_{\mathrm{w}}(\AA)$ & $\lambda_{\max }^{\mathrm{em} a}(\mathrm{~nm})$ & $\lambda_{\max }^{\mathrm{em}}(\mathrm{nm})$ & $\phi_{\mathrm{f}}$ & $\left\langle\tau_{\mathrm{f}}\right\rangle(\mathrm{ns})$ & $k_{\mathrm{r}}\left(\mathrm{s}^{-1}\right) \times 10^{-8}$ & $k_{\mathrm{nr}}\left(\mathrm{s}^{-1}\right) \times 10^{-8}$ & $E_{\mathrm{T}}(30)^{b}$ \\
\hline$w_{\mathrm{o}}=0$ & & 533 & 580 & 0.53 & 3.73 & 1.42 & 1.26 & 49.7 \\
\hline$w_{0}=4$ & 8 & 540 & 625 & 0.32 & 3.62 & 0.88 & 1.88 & 51.6 \\
\hline$w_{0}=8$ & 16 & 540 & 635 & 0.29 & 3.09 & 0.93 & 2.30 & 52.5 \\
\hline$w_{\mathrm{o}}=16$ & 32 & 540 & 640 & 0.23 & 2.50 & 0.93 & 3.06 & 53.8 \\
\hline$w_{\mathrm{o}}=32$ & 64 & 540 & 640 & 0.21 & 2.06 & 1.02 & 3.83 & 54.9 \\
\hline water & & 550 & 665 & 0.018 & 0.65 & 0.28 & 15.11 & 61.3 \\
\hline
\end{tabular}

${ }^{a}$ Obtained from the difference spectra in the case of reverse micelles. ${ }^{b}$ Calculated using the fitting parameters of $\ln \left(k_{\mathrm{nr}}\right)$ vs $E_{\mathrm{T}}(30)$ of the data in Table 1 of ref 11 .
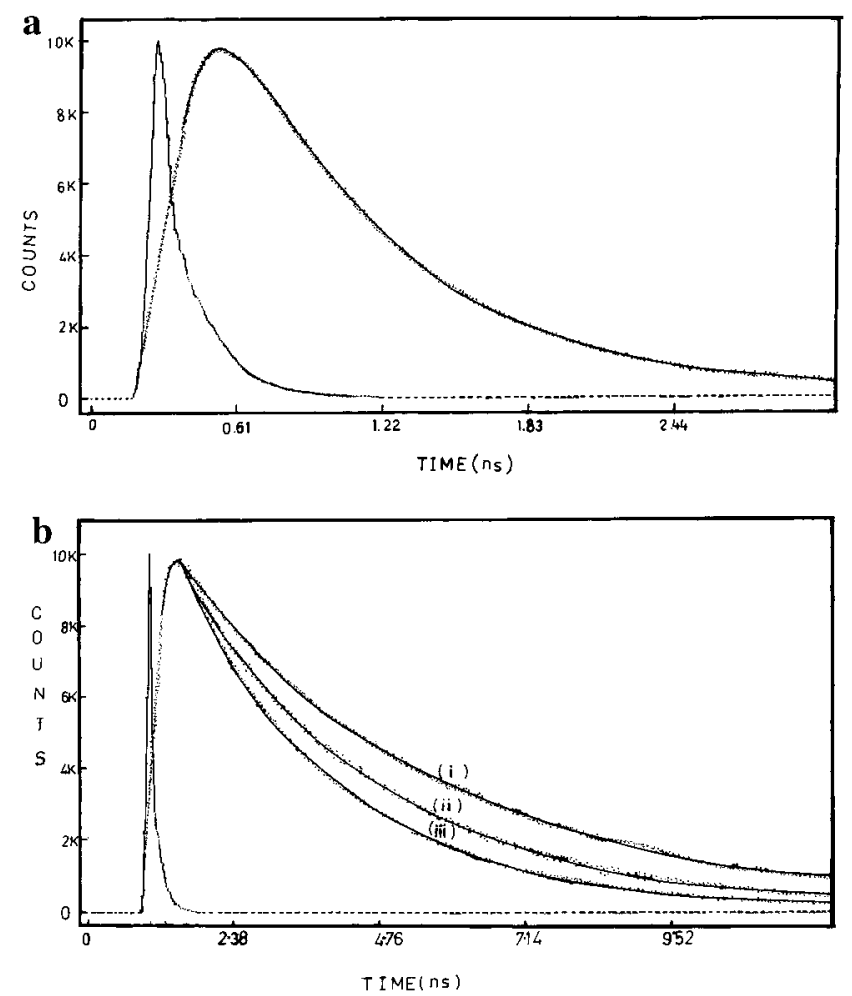

Figure 3. (a) Decay of nile red in water $\left(\lambda_{\mathrm{ex}}=570 \mathrm{~nm}, \lambda_{\mathrm{em}}=660\right.$ $\mathrm{nm})$. The peak near time zero denotes system response. (b) Decay of nile red in $0.09 \mathrm{M}$ AOT in $n$-heptane: (i) $w_{\mathrm{o}}=0$, (ii) $w_{\mathrm{o}}=8$, and (iii) $w_{\mathrm{o}}=32$. The peak near time zero denotes system response.

TABLE 2: Decay Characteristics of Nile Red in 0.09 M AOT in $\boldsymbol{n}$-Heptane at $630 \mathrm{~nm}$

\begin{tabular}{rcccccc}
\hline$w_{\mathrm{o}}$ & $r_{\mathrm{w}}(\AA)$ & $a_{1}$ & $\tau_{1}(\mathrm{~ns})$ & $a_{2}$ & $\tau_{2}(\mathrm{~ns})$ & $\langle\tau\rangle(\mathrm{ns})$ \\
\hline 0 & & 0.10 & 0.36 & 0.90 & 4.11 & 3.73 \\
4 & 8 & 0.07 & 0.71 & 0.93 & 3.84 & 3.62 \\
8 & 16 & 0.11 & 0.59 & 0.89 & 3.40 & 3.09 \\
16 & 32 & 0.16 & 0.51 & 0.84 & 2.88 & 2.50 \\
32 & 64 & 0.24 & 0.21 & 0.76 & 2.65 & 2.06
\end{tabular}

ps, which is similar to that of ordinary water, and another of 10 ns, which is 1000 times slower compared to that of bulk water. ${ }^{6-8}$ Second, comparing the position of the steady-state absorption or emission maxima of many probe molecules with those in different solvents ${ }^{6 a, 18}$ (or comparing present data on nile red with ref 11), the static dielectric constant $\left(\epsilon_{\mathrm{o}}\right)$ of the water pool of the microemulsions seems to be close to that of alcohol (i.e., $\epsilon_{\mathrm{o}} \approx 30$ ). The solvation dynamics in a medium is controlled by the longitudinal relaxation time, $\tau_{\mathrm{L}}=\left(\epsilon_{\infty} / \epsilon_{\mathrm{o}}\right) \tau_{\mathrm{D}}$, where $\epsilon_{\infty}$ and $\epsilon_{\mathrm{o}}$ are respectively the high-frequency and static dielectric constants, and $\tau_{\mathrm{D}}$, the dielectric relaxation time of the medium. $^{4-6,8}$ If one makes the assumption that $\epsilon_{\infty}$ inside the water pool is same as that of ordinary water (i.e., 5), one immediately expects a component of $(5 / 30) \tau_{\mathrm{D}}$. This leads to solvation times of $1.6 \mathrm{ps}$ and $1.6 \mathrm{~ns}$, corresponding to the $10 \mathrm{ps}$ and $10 \mathrm{~ns}$ dielectric relaxation time, respectively. ${ }^{6-8}$ Thus, the dramatic slowing down of the solvation dynamics inside the water pool corresponds to the $1.6 \mathrm{~ns}$ solvation time inside the water pool and is due to the 1000-fold decrease in the dielectric relaxation time of water in organized media. ${ }^{6 a, 7}$

The ICT process is, however, governed by the static polarity of the medium as discussed by Eisenthal et al. ${ }^{13}$ In this case the solvent affects the dynamics of the ICT process by modifying the activation barrier for the ICT process due to the differential stabilization of the "nonpolar" reactant and the polar product (TICT) state or more precisely due to the differential stabilization of the reactant and the transition state (TS). ${ }^{12,13}$ Since during the twisted ICT process the nile red molecule goes from a nearly nonpolar state to a highly polar product, the TS is more polar than the reactant. The preferential solvation of the more polar TS compared to the reactant causes a decrease in the activation barrier of the twisted ICT process with rise in polarity of the medium. ${ }^{12,13}$ The retardation of the ICT process of nile red inside the water pool is, thus, ascribed to the lower static polarity of the water pool compared to that of bulk water.

\section{Conclusions}

The present work demonstrates that the dynamics of the ICT process of nile red inside the water pool of AOT $/ n$-heptane microemulsions are slower by a factor of 8 at $w_{\mathrm{o}}=4$ (corresponds to $r_{\mathrm{w}}=8 \AA$ ) and by a factor of 4 at $w_{\mathrm{o}}=32\left(r_{\mathrm{w}}\right.$ $=64 \AA$ ) compared to that in bulk water. The retardation of the ICT process is attributed to the lower static polarity of the water pool compared to that of bulk water. While the dielectric relaxation behavior of the water molecules in such systems reveals a component 1000 times slower compared to that of bulk water, the static dielectric constant decreases only about 2 times. Since the 2-fold decrease in the static dielectric constant corresponds to a small decrease in $E_{\mathrm{T}}(30)$ values from 63.6 in bulk water to about 50 in reverse micelles and since the rate of the ICT process decreases exponentially with $E_{\mathrm{T}}(30)$, only a 4-8-fold decrease in the rate of the ICT process is observed.

Acknowledgment. Thanks are due to the Department of Science and Technology and Council of Scientific and Industrial Research (CSIR), Government of India, for generous research grants. CSIR is also thanked for providing fellowship to A.D., D.M., and S.K.P.

\section{References and Notes}

(1) (a) Eisenthal, K. B. Chem. Rev. (Washington, D.C.) 1996, 96, 1343. (b) Shen, Y. R. Annu. Rev. Phys. Chem. 1989, 40, 327. (c) Higgins, D. A.; Corn, R. M. Chem. Rev. (Washington, D.C.) 1994, 94, 107.

(2) (a) Zhu, S.-B.; Singh, S.; Robinson, G. W. Adv. Chem. Phys. 1994, 85, 627. (b) Cho, C. H.; Chung, M.; Lee, J.; Nguyen, T.; Singh, S.; Vedamuthu, M.; Yao, S.; Zhu, S.-B.; Robinson, G. W. J. Phys. Chem. 1995, 99, 7806.

(3) (a) Bessho, K.; Uchida, T.; Yamauchi, A.; Shiyoa, T.; Teramae, N. Chem. Phys. Lett. 1997, 264, 381. (b) Yanagimachi, M.; Tamai, N.; Masuhara, H. Chem. Phys. Lett. 1992, 100, 469.

(4) Vajda, S.; Jimenez, R.; Rosenthal, S.; Fiddler, V.; Fleming, G. R.; Castner Jr., E. J. Chem. Soc., Faraday Trans. 1995, 91, 867. 
(5) Lundgren, J. S.; Heitz, M. P.; Bright, F. V. Anal. Chem., 1995, 67, 3775 .

(6) (a) Das, S.; Datta, A.; Bhattacharyya, K. J. Phys. Chem. A 1997, 101, 3299. (b) Sarkar, N.; Das, K.; Datta, A.; Das, S.; Bhattacharyya, K. J. Phys. Chem. 1996, 100, 10523. (c) Sarkar, N.; Datta, A.; Das, S.; Bhattacharyya, K. J. Phys. Chem. 1996, 100, 15483.

(7) (a) Urry, D. W.; Peng, S.; Xu, J.; McPherson, D. T. J. Am. Chem. Soc. 1997, 119, 1161. (b) Bolton, P. S. J. Phys. Chem. 1995, 99, 17061. (c) Fukazaki, M.; Miura, N.; Shiyasiki, N.; Kunita, D.; Shiyoya, S.; Haida, M.; Mashimo, S. J. Phys. Chem. 1995, 99, 431. (d) Mashimo, S.; Kuwabara, S.; Yagihara, S.; Higasi, K. J. Phys. Chem. 1987, 91, 6337. (e) Pethig, R. In Protein-Solvent Interactions; Gregory, R. B., Ed.; Marcel-Dekker: New York, 1995; p 265.

(8) (a) Nandi, N.; Bagchi, B. J. Phys. Chem., 1996, 100, 13914. (b) Nandi, N.; Bagchi, B. Private communication. (c) Bagchi, B.; Chandra, A. Adv. Chem. Phys. 1990, 80, 1. (d) Maroncelli, M. J. Mol. Liq. 1993, 57, 1. (9) (a) Benjamin, I. Chem. Rev. (Washington, D.C.) 1996, 96, 1449. (b) Carpenter, I. L.; Hehre, W. J. J. Phys. Chem. 1990, 94, 531. (d) Rossky, P. J.; Lee, S. H. Chem. Scr. 1989, 29A, 93. (d) Quist, P. O.; Halle, B. J. Chem. Soc., Faraday Trans. 1 1988, 84, 1033.

(10) (a) Sacket, D. L.; Knutson, J. R.; Wolff, J. J. Biol. Chem. 1990 265, 14899. (b) Sacket, D. L.; Wolff, J. Anal. Biochem. 1989, 167, 228. (11) Das, K.; Sarkar, N.; Nath, D.; Bhattacharyya, K. Langmuir 1994, 10,326 .

(12) (a) Rotkiewicz, K.; Grellman, K. H.; Grabowski, Z. R. Chem. Phys. Lett. 1973, 19, 315. (b) Grabowski, Z. R. Pure Appl. Chem. 1993, 65, 1751. (c) Zachariasse, K. A.; von der Haar, T.; Hebecker, A.; Leinhos, U.; Kühnle, W. Pure Appl. Chem. 1993, 65, 1745. (d) Bhattacharyya, K.; Chowdhury, M. Chem. Rev. (Washington, D.C.) 1993, 93, 507.

(13) Hicks, J. M.; Vandersall, M.; Babarogic, Z.; Eisenthal, K. B. Chem. Phys. Lett. 1985, 116, 18.

(14) (a) Luisi, P. L. Angew. Chem., Int. Ed. Engl. 1985, 24, 439. (b) Fendler, J. H. Annu. Rev. Phys. Chem. 1984, 35, 137. (c) Cassin, G.; Badiali, J. P.; Pileni, M. P. J. Phys. Chem. 1995, 99, 12941. (d) Moulik, S. P.; Mukherjee, K. Proc. Indian Natl. Acad. Sci. 1996, 62A, 215.

(15) (a) Zulauf, M.; Eicke, H.-F. J. Phys. Chem. 1979, 83, 480. (b) Jain, T. K.; Varshney, M.; Maitra, A. J. Phys. Chem. 1989, 93, 7409. (c) Maitra, A. J. Phys. Chem. 1984, 88, 5122.

(16) (a) Kotlarchyk, M.; Huang, J. S.; Chen, S.-H. J. Phys. Chem. 1985 89, 4382. (b) Mukherjee, K.; Mukherjee, D. C.; Moulik, S. P. Langmuir 1993, 9, 1727. (c) Jean, Y. C.; Ache, J. H. J. Am. Chem. Soc. 1978, 100, 984, 6320. (d) Eastoe, J.; Young, W. K.; Robinson, B. H. J. Chem. Soc., Faraday Trans. 1990, 86, 2883.

(17) (a) Toptygin, D.; Svobodova, J.; Konopasek, I.; Brand, L. J. Chem. Phys. 1992, 96, 7919. (b) James, D. R.; Ware, W. R. Chem. Phys. Lett. 1985, 120,485 .

(18) (a) Wong, M.; Thomas, J. K.; Gratzel, M. J. Am. Chem. Soc. 1976, 98, 2391. (b) Belletete, M.; Lachapelle, M.; Durocher, G. J. Phys. Chem. 1990, 94, 5337. (c) Guha Ray, J.; Sengupta, P. K. Chem. Phys. Lett. 1994 $75,230$.

(19) Reichardt, C. Chem. Rev. (Washington, D.C.) 1994, 94, 2319.

(20) Kubin, R. P.; Fletcher, A. N. J. Lumin. 1982, 27, 455. 